\title{
The postdictive effect of choice reflects the modulation of attention on choice
}

\author{
Mowei Shen, Yiling Zhou, Luo Chen, Jifan Zhou, Hui Chen
}

Department of Psychology and Behavioral Sciences,

\author{
Zhejiang University, China
}

Data and methods were posted on the Open Science Framework: https://osf.io/r9hb6/

Running head: The impact of attention on choice decision

Word count: 4906 words

Correspondence concerning this article should be addressed to

Hui Chen and Jifan Zhou, Department of Psychology and Behavioral Sciences,

Zhejiang University, Xixi Campus, 148 Tianmushan Road, Hangzhou, 310028,

China,

Telephone number: 0571-88273337, Email: chenhui@zju.edu.cn or

jifanzhou@zju.edu.cn

This work was supported by grants from National Natural Science Foundation of China (No.31771201), National Science Foundation for Distinguished Young Scholars of Zhejiang Province, China (No. LR19C090002), Humanities and Social Sciences Foundation of the Ministry of Education of China (No.17YJA190001) awarded to author Hui Chen. 
Our conscious perception of the world is not an instantaneous, moment-by-moment construction. Rather, our perception of an event is influenced, over time, by information gained following the event: this is known as a postdictive effect. A recent study reported that this postdictive effect could occur even in choice. The present study sought to test whether the striking postdictive effect of choice reflects the modulation of attention on choice, by directly and systematically manipulating attention in two experiments. Specifically, Experiment 1 revealed that the robust postdictive effect of choice was almost completely eliminated when attentional bias was removed. More importantly, Experiment 2 demonstrated that the postdictive effect of choice could be modulated by directly manipulating participants' attention with a spatial cue, in particular, when the cue appeared at short time delays. These results suggest that choice could be considerably postdictively influenced by attention and this effect was most pronounced within a short time window wherein decision-making was most likely in progress. The current study not only enables clarification of the mechanism of the newly discovered postdictive effect of choice, but also extends evidence of the modulation of attention on decision-making.

Keywords: postdictive effect; choice; attention 


\section{Introduction}

Numerous psychological and economic studies have demonstrated that multiple factors can have an impact on choice, such as past experience (Kwon, \& Adaval, 2018; Jahfari, Theeuwes, \& Knapen, 2020; Weiss-Cohen, Konstantinidis, Speekenbrink, \& Harvey, 2018), unconscious thought (Nieuwenstein et al., 2015), motivation (Xin, Xu, Aleman, Luo, \& Feng, 2020; Zhang, \& Feng, 2019), and cognitive load (Deck, \& Jahedi, 2015; Vogels, Krahmer, \& Maes, 2015). Recent studies have shown that choice can even be postdictively biased by an event presented later (e.g., a circle turning red) (Bear \& Bloom, 2016; Bear, Fortgang, Bronstein, \& Cannon, 2017). This postdictive effect is considered a type of illusion as participants mistakenly perceived that they had made a choice before the later event (Bear \& Bloom, 2016). Such a striking postdictive illusion of choice provided initial evidence that experience of choice can be postdictively influenced in a similar manner with lower-level types of visual perception, such as apparent motion and flash-lag effect (Eagleman \& Sejnowski, 2000; Sun, Frank, Hartstein, Hassan, \& Peter, 2017), and higher-level perception such as causal perception and emotional processes (Choi \& Scholl, 2006; Sasaki, Yamada, \& Miura, 2015).

In Bear \& Bloom's (2016) Experiment 1, participants were presented with five identical white circles and instructed to randomly guess which of the circles would turn red. After a variable delay ranging from $50 \mathrm{~ms}$ to $1000 \mathrm{~ms}$, one of the circles turned red, and participants indicated whether they had correctly chosen the circle that turned red, had chosen an incorrect circle, or had not had enough time to make a choice before the circle turned red. The results showed that participants were more likely to report having chosen the red circle, believing that they had made the choice before the circle, which was randomly selected in any given trial, turned red. Crucially, this bias was prominent in short time delay conditions (a delay of roughly $250 \mathrm{~ms}$ or less, wherein decisionmaking is most likely in progress so that the color change of the circle could bias participants' choice before they become consciously aware of the circle turning red), 
and then steadily decreased as the time delay lengthened (wherein decision-making was mostly completed before the unconscious processing of the circle turning red). This bias effect was termed an illusory postdictive effect of choice. Notably, the timedependent characteristic of the postdictive effect suggests that the effect is not simply driven by any general response biases (such as lying) that would exist for all time delay conditions. The postdictive effect was then replicated and extended to a case where there were only two choice options so that the chance level was 0.5 instead of 0.2 as in Experiment 1, which ensured that the observed effect could not be explained by low confidence or random responding. Specifically, participants were required to mentally select one of the two different-colored circles. After a variable delay, a new circle, which matched the color of one of the two circles, appeared in the center of the screen. Participants were asked to indicate whether or not the new circle matched the color of the circle they had chosen. The results showed a clear postdictive effect, even for the binary choice task. Interestingly, in a follow-up study this counterintuitive postdictive illusion of choice was found to be greater for participants who were more prone to delusions (Bear et al., 2017).

Clearly, the postdictive illusion of choice reported above was a robust effect that persisted across different paradigms and populations. However, the mechanism underlying this effect remains unexplored. It should be noted that although Bear and Bloom (2016) had proposed that a circle turning red too soon (while decision-making was still in progress) might bias participants' choice unconsciously through subliminally capturing visual attention (Chen \& Wyble, 2018; Yantis \& Jonides, 1984, 1990), their study was not able to test this possibility because attention was not directly manipulated. Furthermore, as they acknowledged, it is also possible that participants had completed their choices but not fully encoded them into memory before the circles turned red. In this perspective, the postdictive illusion of choice might reflect the fact that participants' memories of choices were modified by the subsequent events (circles turning red). Therefore, the current study sought to test whether the novel postdictive effect of choice reflects the modulation of attention on choice by employing a modified 
version of the aforementioned postdictive paradigm. More importantly, the current study enables clarification of the mechanism of the newly discovered postdictive effect of choice, which fills in the gaps left by Bear \& Bloom's (2016) study.

The current study contained two experiments. Experiment 1 was designed on the basis of Bear and Bloom's study (2016) but eliminated participants' attentional bias to the red circle, to test whether the observed postdictive effect of choice would be absent after the influence of attention was controlled. Experiment 2 employed a combination of Bear and Bloom's (2016) postdictive paradigm and the classic spatial cueing paradigm (Chen \& Wyble, 2018; Posner, 1980; Posner, Snyder, \& Davidson, 1980), to seek more direct evidence of the impact of attention on the postdictive effect of choice.

\section{Experiment 1}

We modified Bear and Bloom's (2016) Experiment 1 by presenting participants with two white circles and then suddenly changing the colors of both circles simultaneously, so that participants' attention would no longer be automatically biased to any one particular circle.

\section{Method}

Participants. This study closely followed Bear and Bloom's (2016) procedure. Based on their study, we predicted a large effect size (odds ratio $=1.59 * 10^{11}$ ) for our experimental design and performed a power analysis in $\mathrm{G}^{*}$ power 3 (Faul, Erdfelder, Buchner, \& Lang, 2009), which determined that with 95\% power at an $\alpha$ level of .05, the sample size needed to achieve the predicted effect size was appropriately 17 individuals. We thus used a predetermined sample size of 25 participants, in line with Bear and Bloom's (2016) study. Twenty-six undergraduate students from Zhejiang University completed Experiment 1 for either course credit or money. One participant was excluded because he correctly guessed the purpose of the experiment when he was debriefed after the experiment, leaving 25 participants for inclusion in the final analyses 
132

133

134

135

(16 females, 9 males; mean age $=19.20)$. Notably, the results pattern did not change even when the excluded participant was included. Informed consent was obtained from each participant prior to each study in accordance with the institutional review board at Zhejiang University. All participants had normal, or corrected-to-normal vision and had no color vision defects.

Apparatus. The stimuli were presented on a 17-inch CRT display $(1,024 \times 768$ pixels; $60 \mathrm{~Hz}$ ) with the background color set to black (RGB: 0, 0, 0). Participants viewed the stimuli at a distance of approximately 50 centimeters. The experiment was run in MATLAB with the Psychophysics Toolbox (Brainard, 1997; Pelli, 1997). Responses were recorded by a computer keyboard.

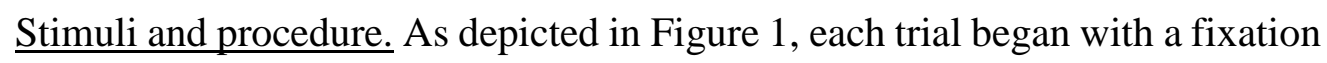
cross $\left(0.62^{\circ}\right)$ in the center of the screen for 500 milliseconds, followed by two identical white circles $\left(2.01^{\circ} \times 2.01^{\circ}\right.$; RGB: $\left.255,255,255\right)$. Both circles were positioned at two random locations on the circumference of an imaginary circle (radius $=6.04^{\circ}$ ) centered on the screen, with a constraint that the two circles were separated from each other at least by $6.04^{\circ}$ (center-to-center). Participants were instructed to mentally choose one of two circles once they were presented. After a variable delay of 50.00, 83.33, 166.67, 250.00, 333.33, 500.00, or 1,000.00 ms, one randomly selected circle turned red (RGB: $255,0,0)$ while the other circle turned a different color that was randomly chosen from a set of five colors: gold (RGB: 255, 215, 0), green (RGB: 0, 255, 0), fuchsia (RGB: 255, 0, 255), aqua (RGB: 0, 255, 255), and coral (RGB: 255, 127, 80). Participants were asked to press a corresponding key to indicate whether they had chosen the circle that turned red (press "Y" for "yes"), had chosen another circle (press "N" for "no"), or had not had enough time to make a choice before they noticed the occurrence of the color change (press "D" for "did not have enough time"). The colored circles remained on the screen until a response was emitted. 


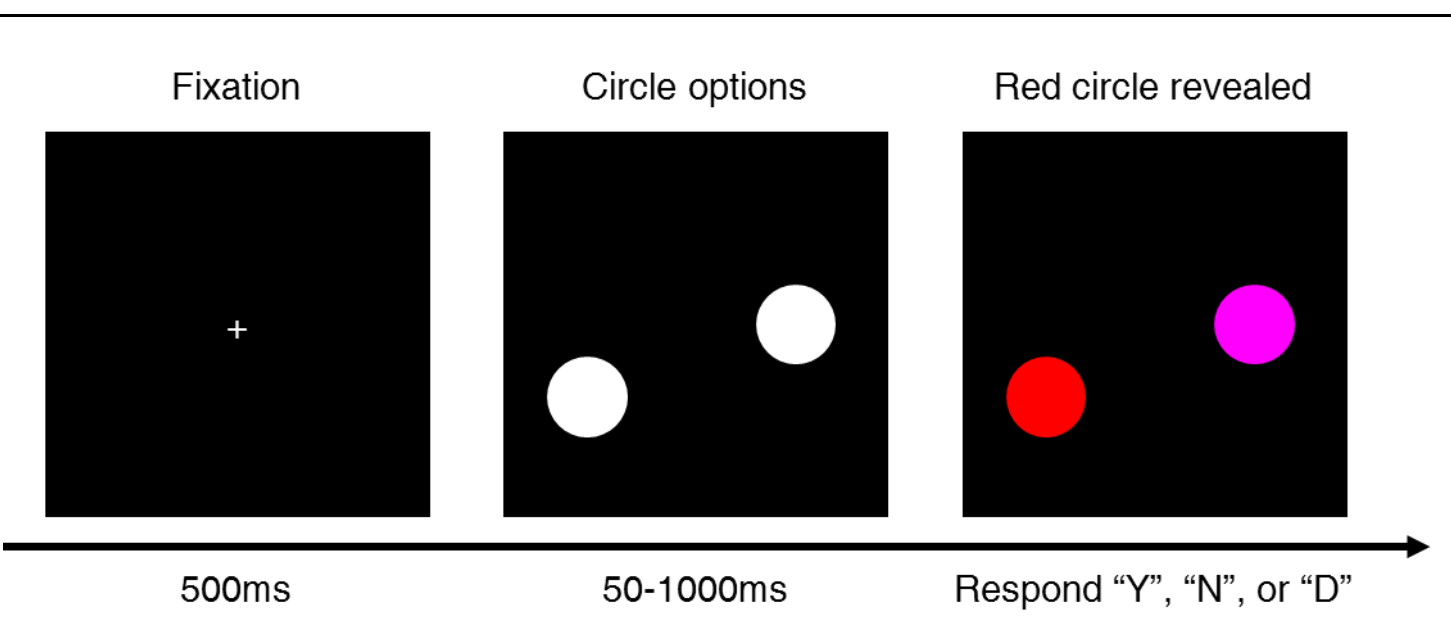

Figure 1. Experimental procedure of Experiment 1. After fixation, two white circles appeared in random positions on an imaginary circle, and participants were asked to mentally choose one of the circles. After a variable delay, both circles then changed color, one turned red and the other turned a different color. While these circles remained on the screen, participants pressed a key on the keyboard to indicate whether they had chosen the circle that turned red ("Y"), had chosen a different circle ("N"), or had not had enough time to make a decision before the circles changed color ("D”).

All participants completed 5 blocks, with each block containing 56 trials divided equally into 7 time-delay conditions, resulting in a total of 280 trials. The trials of different conditions were randomly ordered within each block. Participants completed 14 practice trials before the experimental trials began. After the experiment, all participants were debriefed as to whether they had used any strategies or had guessed the purpose of the experiment. participants reported that they had not had enough time to make a choice before they noticed the occurrence of the color change, were excluded from analysis, with $14.9 \%$ of trials being removed. The removed trials for each condition were as follows: 50.00 ms: 37.9\%, 83.33 ms: 33.3\%, $166.67 \mathrm{~ms}: 18.3 \%, 250.00 \mathrm{~ms}: 7.5 \%, 333.33 \mathrm{~ms}: 5.3 \%$, $500.00 \mathrm{~ms}: 1.7 \%$, and $1000.00 \mathrm{~ms}: 0.5 \%$. 
We used a logistic regression, which was also applied by Bear and colleagues (Bear \& Bloom, 2016; Bear et. al., 2017), to analyze the postdictive effect of choice, that is, whether the probability of choosing the red circle varied with the log of time delay (measured in milliseconds). The regression was performed using logit command in Stata 16 SE (StataCorp., 2019). Specifically, the log of time delay was modeled as an independent variable. Standard errors were clustered on the participant variable to account for the non-independence of repeated observations from the same participant. The dependent variable was whether or not participants had chosen the red circle. In this regression, the coefficient of the time delay variable indicates how often participants are likely to report having chosen the red circles. In other words, if the coefficient is significantly greater than 0 , it will suggest that the likelihood of choosing the red circle decreases as the time delay gets longer (i.e., a postdictive effect was observed).

\section{$\underline{\text { Results and Discussion }}$}

The results of this experiment are depicted in Figure 2. The analysis showed that the regression was not significant, model $\chi^{2}(1)=0.58, p=.446$. The effect of time delay was also not significant, $\beta=-0.02,95 \%$ CI $[-.09, .04], z=-0.76, p=.446$, suggesting that the probability of choosing the red circle did not significantly vary with the time delay, namely, the postdictive effect was not observed. Bayesian analysis confirmed the absence of a postdictive effect. The Bayes factor in favor of the alternative hypothesis that $\beta$ is different from zero versus the null hypothesis that $\beta$ is zero was $\mathrm{BF}_{10}=.0004$ provides anecdotal evidence for the alternative hypothesis. 


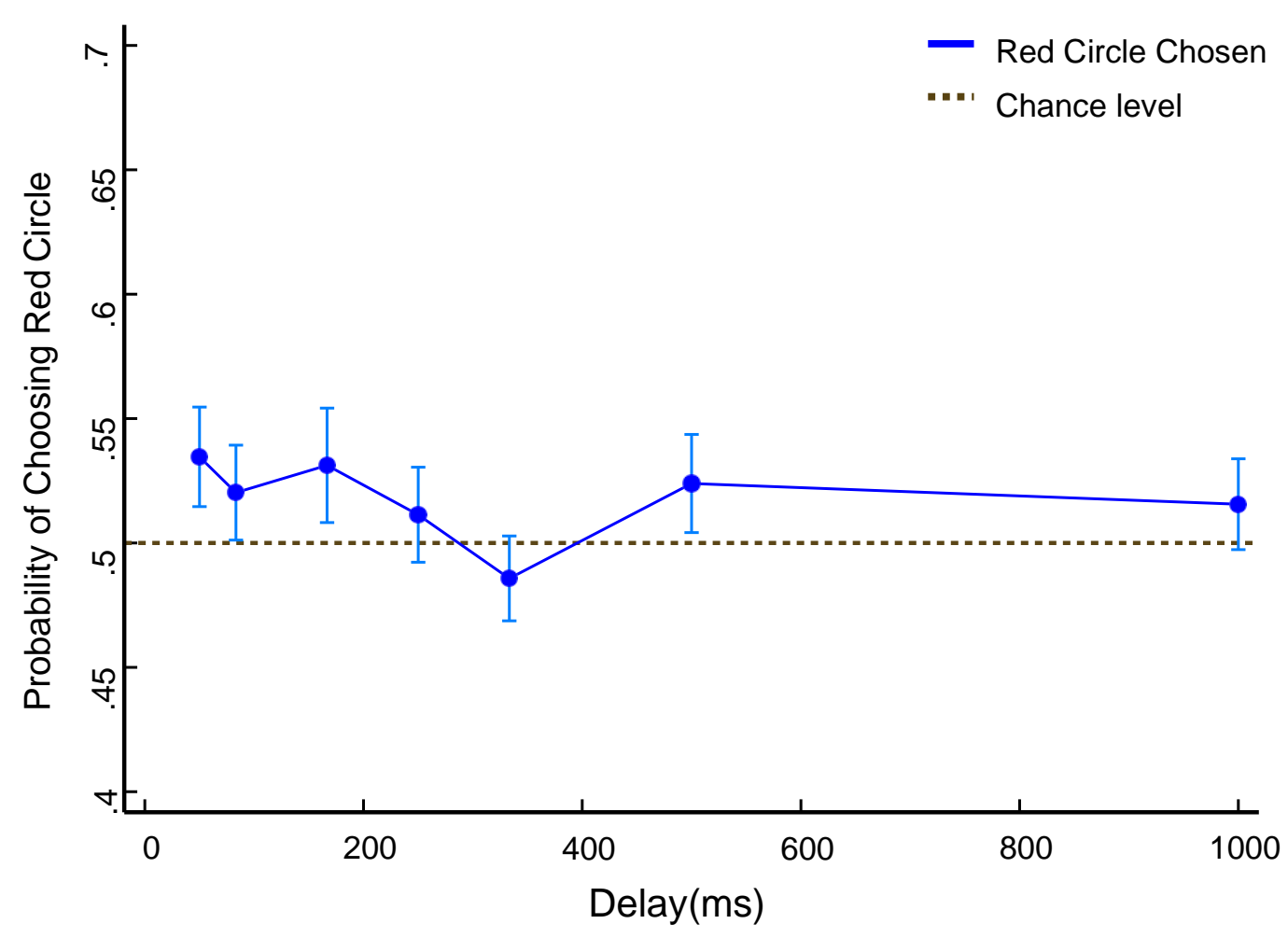

202 Figure 2. Results from Experiment 1. The blue line shows the probability that participants chose 203 the red circle in trials in which they claimed to have had enough time to make a choice. The 204 error bars denote $95 \%$ confidence intervals. The dotted line represents the chance level.

As expected, the results of Experiment 1 showed that the postdictive effect reported by Bear and Bloom (2016) almost disappeared when participants' attention was no longer automatically captured by the circle that turned red. Therefore, the observed postdictive effect in Bear and Bloom (2016) was mainly, if not entirely, driven by an automatic attentional capture to the sudden color change of the circle.

\section{Experiment 2}

To further confirm that this postdictive effect reflects the effect of attention

214 on choice, we modified Experiment 1 by directly manipulating attention with the use

215 of a classical spatial cue (Chen \& Wyble, 2018; Posner, 1980; Posner et al., 1980). 


\section{Method}

This experiment was identical to Experiment 1 with the following exceptions (see Figure 3). Twenty-eight new participants completed Experiment 2, with three participants excluded by the criterion described previously (16 females, 9 males; mean age $=19.08)$. Notably, the results pattern did not change even with the inclusion of the excluded participants. As the two white circles changed colors, a spatial cue was simultaneously presented and remained on the screen for $100 \mathrm{~ms}$. The cue consisted of two identical white rectangle bars $\left(0.20^{\circ} \times 2.01^{\circ}\right)$, with each one being presented $0.20^{\circ}$ above and below one of the circles. In half of the trials, the cue had the same location as the red circle, which was termed a valid condition ${ }^{1}$. Whereas in the other half of the trials, the cue was located at the position of another colored circle, which was termed an invalid condition. Participants were explicitly instructed that the cue was uninformative about which circle would turn red and thus should be ignored.

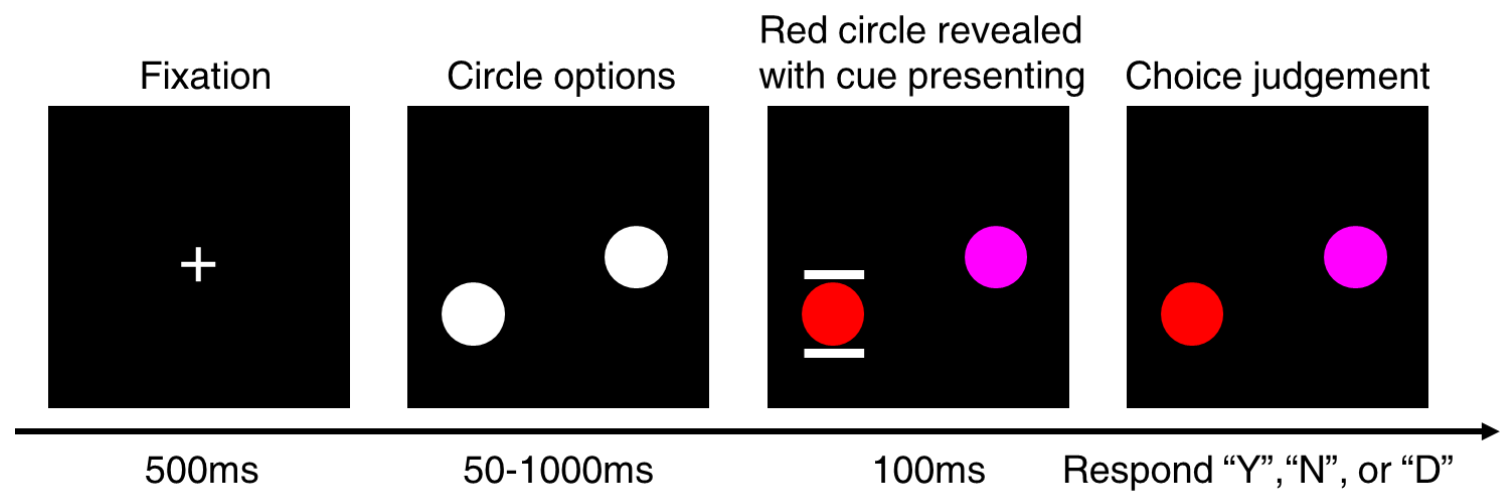

231 Figure 3. Experimental procedure in Experiment 2. After fixation, two white circles appeared 232 in random positions, and participants were asked to mentally choose one of the circles. After a 233 variable delay, both circles then changed colors, one turned red and the other turned a different 234 color. Meanwhile, a cue was also briefly presented either with the red circle (valid trials) or the

\footnotetext{
${ }^{1}$ Note that the valid and invalid conditions here were not essentially the same as those in spatial cueing studies. We used these terms (valid and invalid) just for ease of description and understanding.
} 
other circle (invalid trials). While these circles remained on the screen, participants pressed a key on the keyboard to indicate whether they had chosen the circle that turned red ("Y"), had chosen a different circle ("N"), or had not had enough time to reach a decision before the circles changed colors ("D").

The experiment adopted a 2 cue validity (valid and invalid) $\times 7$ time-delay $(50.00,83.33,166.67,250.00,333.33,500.00$, or $1,000.00 \mathrm{~ms})$. There were 40 trials for each of the 14 conditions, resulting in a total of $560(40 \times 14)$ trials equally divided into 8 blocks. The trials of different conditions were randomly ordered within each block. Participants completed 14 practice trials before the experiment. As in Experiment 1, all participants were debriefed after the experiment task.

Data analysis. As in Experiment 1, the trials where participants reported that they had not had enough time to make a choice before they noticed the occurrence of the color change were excluded from analysis, with $8.54 \%$ of trials being removed. The removed trials for each condition were as follows: $50.00 \mathrm{~ms}: 23.3 \%, 83.33 \mathrm{~ms}: 18.0 \%$, $166.67 \mathrm{~ms}: 9.1 \%, 250.00 \mathrm{~ms}: 4.9 \%, 333.33 \mathrm{~ms}: 2.2 \%, 500.00 \mathrm{~ms}: 1.8 \%$, and 1000.00 ms: $0.5 \%$ for $50.00,83.33,166.67,250.00,333.33,500.00$, or $1,000.00 \mathrm{~ms}$.

In order to assess whether the postdictive effect could be yielded by attention triggered by a cue, as in Experiment 1, we ran a logistic regression to analyze whether the probability of choosing the red circle varied with the log of time delay (measured in milliseconds). The log of time delay and validity of the cue, along with the interaction between the log of time delay and validity were modeled as independent variables. Standard errors were clustered on the participant variable to account for the nonindependence of repeated observations from the same participant.

\section{$\underline{\text { Results and discussion }}$}

A model comparison revealed that this interaction model (Akaike Information 
Criterion, $\mathrm{AIC}=17686.45$ ) was preferable to a model where the interaction term was not included $(\mathrm{AIC}=17693.75)$. Furthermore, the interaction model of this logistic regression was significant, $\chi^{2}(3)=22.28, p<.001$. A significant interaction between validity of the cue and time delay was also observed, $\beta=0.12,95 \%$ CI $[.04, .19], z=$ $3.07, p=.002$, indicating that the probability of reporting having chosen the red circle as a function of the delay (i.e., postdictive effect) differed between two different validity conditions. In an attempt to further explain this interaction effect, we ran logistic regressions for the valid and invalid conditions (see Figure 4). In the valid condition, the rate of choosing the red circle increased significantly as the time delay decreased, $\beta$ $=-0.10,95 \%$ CI $[-.16,-.04], z=-3.31, p=.001\left(\operatorname{model} \chi^{2}(1)=10.98, p<.001\right)$, indicating that the postdictive effect of choice was obtained. Whereas in the invalid condition, there was almost no postdictive effect, $\beta=.01,95 \%$ CI $[-.05, .07], z=0.45$, $p=.651($ model $\chi 2(1)=0.21, p=.651)$. Bayesian analysis confirmed the absence of a postdictive effect in the invalid condition $(\mathrm{BF} 10=.0003)$.

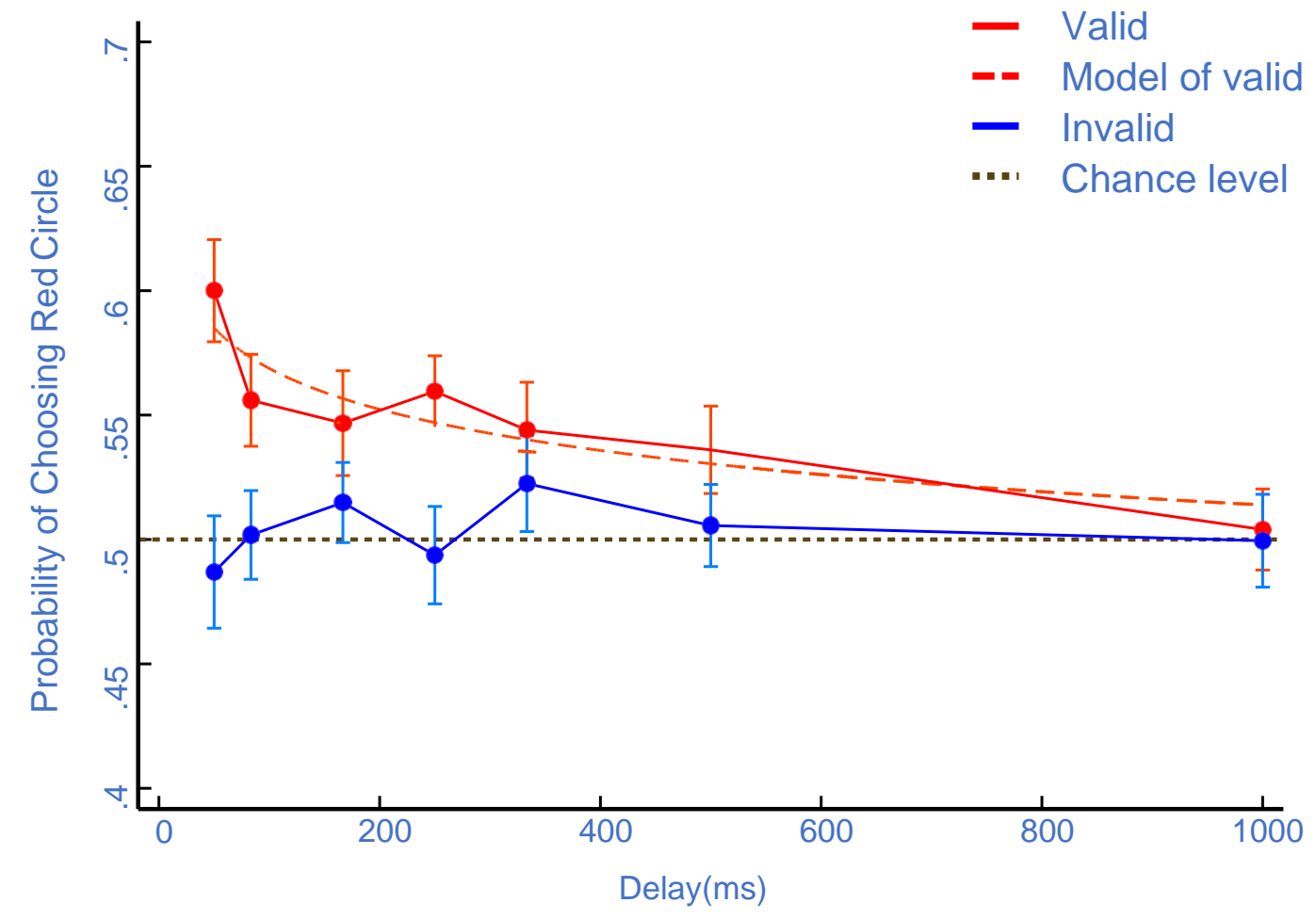


Figure 4. Results from Experiment 2. The red line and blue line show the probability that participants chose the red circle in trials in which they claimed having had enough time to make a choice for valid and invalid trials, respectively. The red dotted line shows the result of the best-fitting logistic model of responses as a function of the time delay in the valid condition. The brown dotted line represents the chance level.

The results of Experiment 2 revealed a postdictive effect in the valid condition, in that directing participants' bottom-up attention to the red circle with a spatial cue increased the probability of claiming to have chosen that circle, particularly with the shorter time delay. However, in the invalid condition we did not obtain a "reverse" postdictive effect, which, had there been any, would have revealed a similar but reversed pattern of the valid condition (the bias to choose the red circle would have been smallest for the shortest delays and would have increased with an increase in the delay). We postulated that the absence of this postdictive effect might be due to the fact that the attentional capture of the salient spatial cue, which suddenly appeared at the location of the other non-red circle, was counteracted by the top-down attentional bias toward the red circle. The top-down attentional bias occurred because participants were asked to indicate whether or not they had chosen the red circle, which made the red circle more relevant to the participants' task goal. Another possibility might be that attentional bias to the red color was because red was the salient feature being referenced, which means that the same bias would have occurred even if the goal had been to "avoid" picking the red circle. ${ }^{2}$ Notably, such a top-down attentional bias alone was not sufficient to elicit the postdictive effect of choice, as revealed by an absence of this postdictive effect in Experiment 1. In conclusion, the results of Experiment 2 provided stronger and more direct evidence of the critical contribution of attention in yielding the postdictive effect of choice. 


\section{General Discussion}

306

307

308

309

310

311

312

313

314

The present study, based on Bear and Bloom's research (2016), sought to test whether the striking postdictive effect of choice reflects the modulation of attention on choice, by manipulating attention systematically and directly. Specifically, Experiment 1 revealed that the robust postdictive effect of choice was almost completely eliminated when the attentional bias triggered by a sudden color change was removed. More importantly, Experiment 2 demonstrated that the postdictive effect of choice could be modulated by directly manipulating participants' attention with a spatial cue. These findings provide converging evidence that simple choice could be postdictively modulated by attention.

\section{Implications for understanding the postdictive effect}

Previous studies on postdictive processing suggest that there is a limited temporal window within which the processing of sensory stimuli presented later can affect the perception of already-presented stimuli. For instance, the illusory perception of apparent motion shows that when viewing a rapid succession of two flashes posited at two discrete locations, participants often report seeing a flash moving continuously and smoothly from the first to the second location (Kolers \& von Grünau, 1976). The flash-lag effect shows that when a stationary stimulus is briefly presented physically aligned with a continuously moving object, the position of the stimulus is seen to lag behind the position relative to the moving object (Eagleman \& Sejnowski, 2000). In addition to these low-level simple phenomena, the postdictive effect could also emerge in high-level perceptions like causality perception (Choi \& Scholl, 2006) and emotional processing (Sasaki et al., 2015). Choi and Scholl (2006) demonstrated that participants perceived a collision between two objects (i.e., one ball causing the other to move) in an ambiguous display even after the moment of potential overlap for collision had already passed. Sasaki et al. (2015) found that when participants were required to drag 
a dot up or down on the screen immediately after showing an emotional image, they would rate the image more positively if the dragging action was up and rate it more negatively if the dragging action was down.

The current study, together with that of Bear and Bloom (2016), complements the literature on postdictive perceptual phenomena by providing converging evidence that the experience of choice could also be postdictively biased in a similar way. More importantly, the findings of the current study have critical implications for understanding the mechanism of postdictive effects, which is a highly debated topic in the field (Eagleman, \& Sejnowski, 2000; Van Wassenhove, 2009). Here we focus on two accounts adopted by Bear and Bloom (2016) to explain the postdictive effect of choice: the perceptual interpretation (Bear \& Bloom, 2016; Choi \& Scholl, 2006; Hermens, Luksys, Gerstner, Herzog, \& Ernst, 2008; Rao, Eagleman, \& Sejnowski, 2001; Tye, 2003) and the memory interpretation (Dennett, 1993; Phillips, 2011). The former account suggests participants' choice was unconsciously biased through attentional capture triggered by the color change of a circle happening within the decision-making window, although they believed that the circles turned red after they had made their decisions. In contrast, the memory hypothesis posits that despite participants having initially made choices, they had not had enough time to fully encode the choices into memory before the circles turned red at short time delays, and thus their memories could be easily interfered with or modified by the encoding of subsequent low-level visual changes.

The findings of the present study can hardly be explained by the memory hypothesis. According to this memory-revision account, in the short time delay conditions of Experiment 2, participants' memory of choices could be interfered by two different aspects of visual changes, one was the subsequent presentation of the spatial cue and the other was the color change of two circles. Thus, two possible result patterns could be predicted in this case. First, as both circles had visual changes (regardless of the color change or cue presentation) that would interfere with the memory of choices, participants might have no bias to choose any changed circles and would thus be likely 
to choose one of two circles randomly. If this was true, no postdictive effect should have been observed in both valid and invalid conditions. Second, when memory of choices was not fully encoded, participants might have had a tendency to report the circle where the cue was presented as their initial choice. This possibility would predict a postdictive effect in the valid condition and a reverse postdictive effect in the invalid condition. Clearly, both predictions from the memory-revision explanation were not supported by the results of Experiment 2 which showed a postdictive effect in the valid condition whereas no such effect was observed in the invalid condition.

In contrast, the current study provided direct and strong evidence supporting the perception account focusing on the effect of attention. First, Experiment 1 demonstrated that the postdictive effect of choice was removed when the bottom-up attentional capture (or bias to the red circle) triggered by the sudden color change was controlled/eliminated. Moreover, Experiment 2 (valid condition) showed that the postdictive effect of choice reappeared after orienting participants' attention to the red circle with a spatial cue presenting simultaneously with the color changes of the circles. Interestingly, an unexpected result was observed in the invalid condition in which no postdictive effect was observed. We postulated that this absence of postdictive effect was due to the fact that participants inevitably biased their attention toward the red circle in a top-down manner, which counteracted the effect of bottom-up attentional capture triggered by the sudden appearance of the salient spatial cue at the location of the non-red circle. Consequently, these results support the aforementioned perception account by showing that attention plays a crucial role in driving the postdictive effect of choice. This is consistent with previous studies showing that postdictive effects such as apparent motion and flash-lag effect were significantly modulated by attention (Chappell, Hine, Acworth, \& Hardwick, 2006; Kohler, Haddad, Singer, \& Muckli, 2008; Sun et al., 2017).

\section{Implications for understanding conscious perception}

Although we experience the world smoothly and continuously, there is a long- 
standing debate about whether conscious perception is continuous or discrete. Continuous theories of consciousness propose that consciousness is a continuous stream of percepts. That is, sensory information is continuously translated into conscious perception (e.g., Fekete, Van de Cruys, Ekroll, \& van Leeuwen, 2018). In contrast, discrete theories of consciousness argue that conscious perception is a series of distinct moments, with the information being collected only at discrete points of time (VanRullen, \& Koch, 2003; White, 2018). Postdictive effects, including the one presented in the current study, reflect that the conscious perception of an earlier stimulus or event can be influenced by a later one, which seems to be incompatible with continuous theories but supports discrete theories.

More recently, a two-stage discrete model was proposed to further clarify that perception is discrete (Herzog, Kammer, \& Scharnowski, 2016; Herzog, Drissi-Daoudi, $\&$ Doerig, 2020). This model argued that our brain does not collect information only at certain time points like a camera, instead, information is unconsciously integrated over an extended period of time preceding discrete conscious percepts. The two-stage discrete model has been supported by studies of long-lasting postdictive effects in a variety of paradigms (Drissi-Daoudi, Doerig, \& Herzog, 2019; Sergent et. al., 2013; Sun et al., 2017). The current study provided additional evidence for this model showing that choice can even be influenced by a later event that occurs after a specific time period (several hundreds of milliseconds).

\section{Implications for understanding the effect of attention on decision-making}

Previous work has provided consistent evidence that attention significantly influences decision-making. One of the pioneering studies is by Shimojo, Simion, Shimojo, and Scheier (2003), who presented participants with pairs of human faces and asked them to decide which face was more attractive. They monitored gaze position while participants made choices and found that participants were more likely to choose the face that they looked at the longest. Numerous subsequent studies replicated and 
extended this finding by exploiting different types of decision-making such as moral decisions (e.g., Pärnamets et al., 2015), consumer decisions (e.g., Krajbich, Armel, \& Rangel, 2010), and risky decisions (e.g., Ashby et al., 2018). These findings were usually interpreted as evidence that attention could significantly influence choices, since the items that were looked at the longest, or more frequently, were typically regarded as receiving more attention.

The current study extended previous work in three crucial ways. First, the two choice options were identical artificial items (e.g., circles), rather than different realworld objects (e.g., faces) as in most previous studies, which ensured that participants could not make a choice based on their inherent preferences that were independent of attention. Second, attention was more directly manipulated by using the classic and well-accepted spatial cue paradigm (Chen \& Wyble, 2018; Posner, 1980; Posner et al., 1980), which could overcome the possible limitations associated with eye-tracking based methodology (Gwinn, Leber, \& Krajbich., 2019), and thus provide more direct and causal evidence for the effect of attention in decision-making. Finally, the current study not only tested whether or not (yes or no) attention could affect decision-making, but also provided stringent ways of evaluating this effect by systematically assessing the time window in which the attentional effect on choice decisions can occur. To our knowledge, only one previous study addressed the effect of attention on choice over time (Milosavljevic, Navalpakkam, Koch, \& Rangel, 2012). However, in that study the researchers manipulated the saliency of stimuli (i.e., snacks) to alter attention, which makes it difficult to exclude the potential confounding of response bias. That is, participants may have chosen the more salient stimulus because it was perceived more clearly in the limited exposure time. This issue did not exist in the current study in which two identical simple circles were used as options and participants did not need to recognize the option stimuli during the choice decision.

It is worth mentioning that the choices participants made in our study were largely arbitrary because no costs or rewards were associated with the options, in contrast to much of the literature attempting to connect attention (gaze) and decision- 
making. Thus, future work needs to further address this issue by bridging the gap between the current study and the literature on value-based decision making. Nonetheless, the current study should still provide novel insight in the field of attentional effect on decision-making.

\section{Conclusion}

The current study demonstrated that the striking postdictive effect of choice, discovered recently by Bear and Bloom (2016), reflects the modulation of attention on choice. These results suggest that choice can be considerably postdictively influenced by attention, which has critical implications for the literature on postdictive effects and the effects of attention on decision-making.

\section{Acknowledgements}

This work was supported by grants from National Natural Science Foundation of China (No.31771201), National Science Foundation for Distinguished Young Scholars of Zhejiang Province, China (No. LR19C090002), Humanities and Social Sciences Foundation of the Ministry of Education of China (No.17YJA190001) awarded to author Hui Chen.

\section{References}

Ashby, N. J., Yechiam, E., \& Ben-Eliezer, D. (2018). The consistency of visual attention to losses and loss sensitivity across valuation and choice. Journal of Experimental Psychology: General, 147(12), 1791.

Bear, A., \& Bloom, P. (2016). A simple task uncovers a postdictive illusion of choice. Psychological science, 27(6), 914-922.

Bear, A., Fortgang, R. G., Bronstein, M. V., \& Cannon, T. D. (2017). Mistiming of thought and perception predicts delusionality. Proceedings of the National Academy of Sciences, 114(40), 10791-10796. 
Brainard, D. H. (1997). The psychophysics toolbox. Spatial vision, 10, 433-436.

Chappell, M., Hine, T. J., Acworth, C., \& Hardwick, D. R. (2006). Attention 'capture'by the flash-lag flash. Vision research, 46(19), 3205-3213.

Chen, H., \& Wyble, B. (2018). The neglected contribution of memory encoding in spatial cueing: A new theory of costs and benefits. Psychological Review, 125, 936-968.

Choi, H., \& Scholl, B. J. (2006). Perceiving causality after the fact: Postdiction in the temporal dynamics of causal perception. Perception, 35(3), 385-399.

Deck, C., \& Jahedi, S. (2015). The effect of cognitive load on economic decision making: A survey and new experiments. European Economic Review, 78, $97-$ 119.

Dennett, D. C. (1993). Consciousness explained: Penguin uk.

Drissi-Daoudi, L., Doerig, A., \& Herzog, M. H. (2019). Feature integration within discrete time windows. Nature communications, 10(1), 1-8.

Eagleman, D. M., \& Sejnowski, T. J. (2000). Motion integration and postdiction in visual awareness. Science, 287(5460), 2036-2038.

Faul, F., Erdfelder, E., Buchner, A., \& Lang, A. G. (2009). Statistical power analyses using G* Power 3.1: Tests for correlation and regression analyses. Behavior research methods, 41(4), 1149-1160.

Fekete, T., Van de Cruys, S., Ekroll, V., \& van Leeuwen, C. (2018). In the interest of saving time: a critique of discrete perception. Neuroscience of consciousness, 2018(1), niy003.

Gwinn, R., Leber, A. B., \& Krajbich, I. (2019). The spillover effects of attentional learning on value-based choice. Cognition, 182, 294-306.

Hermens, F., Luksys, G., Gerstner, W., Herzog, M. H., \& Ernst, U. (2008). Modeling spatial and temporal aspects of visual backward masking. Psychological review, 115(1), 83-100.

Herzog, M. H., Drissi-Daoudi, L., \& Doerig, A. (2020). All in Good Time: LongLasting Postdictive Effects Reveal Discrete Perception. Trends in Cognitive Sciences.

Herzog, M. H., Kammer, T., \& Scharnowski, F. (2016). Time slices: what is the duration of a percept?. PLoS Biology, 14(4), e1002433.

Jahfari, S., Theeuwes, J., \& Knapen, T. (2020). Learning in visual regions as support for the bias in future value-driven choice. Cerebral Cortex, 30(4), 2005-2018. 
Kolers, P. A., \& von Grünau, M. (1976). Shape and color in apparent motion. Vision research, 16(4), 329-335.

Kohler, A., Haddad, L., Singer, W., \& Muckli, L. (2008). Deciding what to see: The role of intention and attention in the perception of apparent motion. Vision research, 48(8), 1096-1106.

Krajbich, I., Armel, C., \& Rangel, A. (2010). Visual fixations and the computation and comparison of value in simple choice. Nature neuroscience, 13(10), 1292-1298.

Kwon, M., \& Adaval, R. (2018). Going against the flow: The effects of dynamic sensorimotor experiences on consumer choice. Journal of Consumer Research, 44(6), 1358-1378.

Milosavljevic, M., Navalpakkam, V., Koch, C., \& Rangel, A. (2012). Relative visual saliency differences induce sizable bias in consumer choice. Journal of Consumer Psychology, 22(1), 67-74.

Nieuwenstein, M. R., Wierenga, T., Morey, R. D., Wicherts, J. M., Blom, T. N., Wagenmakers, E. J., \& van Rijn, H. (2015). On making the right choice: A meta-analysis and large-scale replication attempt of the unconscious thought advantage. Judgment and Decision Making, 10(1), 1-17.

Pärnamets, P., Johansson, P., Hall, L., Balkenius, C., Spivey, M. J., \& Richardson, D. C. (2015). Biasing moral decisions by exploiting the dynamics of eye gaze. Proceedings of the National Academy of Sciences, 112(13), 4170-4175.

Pelli, D. G. (1997). The VideoToolbox software for visual psychophysics: Transforming numbers into movies. Spatial vision, 10(4), 437-442.

Phillips, I. B. (2011). Perception and iconic memory: what Sperling doesn't show. Mind \& Language, 26(4), 381-411.

Posner, M. I. (1980). Orienting of attention. Quarterly journal of experimental psychology, 32(1), 3-25.

Posner, M. I., Snyder, C. R., \& Davidson, B. J. (1980). Attention and the detection of signals. Journal of Experimental Psychology: General, 109(2), 160-174.

Rao, R. P., Eagleman, D. M., \& Sejnowski, T. J. (2001). Optimal smoothing in visual motion perception. Neural Computation, 13(6), 1243-1253.

Sasaki, K., Yamada, Y., \& Miura, K. (2015). Post-determined emotion: motor action retrospectively modulates emotional valence of visual images. Proceedings of the Royal Society B: Biological Sciences, 282(1805), 20140690.

Sergent, C., Wyart, V., Babo-Rebelo, M., Cohen, L., Naccache, L., \& Tallon-Baudry, C. (2013). Cueing attention after the stimulus is gone can retrospectively trigger conscious perception. Current biology, 23(2), 150-155. 
Shimojo, S., Simion, C., Shimojo, E., \& Scheier, C. (2003). Gaze bias both reflects and influences preference. Nature neuroscience, 6(12), 1317-1322.

StataCorp. (2019). Stata Statistical Software: Release 16. College Station, TX:

Sun, L., Frank, S. M., Hartstein, K. C., Hassan, W., \& Peter, U. T. (2017). Back from the future: Volitional postdiction of perceived apparent motion direction. Vision Research, 140, 133-139.

Tye, M. (2003). Consciousness and persons: Unity and identity. Cambridge, MA: MIT Press.

VanRullen, R., \& Koch, C. (2003). Is perception discrete or continuous?. Trends in cognitive sciences, 7(5), 207-213.

Van Wassenhove, V. (2009). Minding time in an amodal representational space. Philosophical Transactions of the Royal Society B: Biological Sciences, 364(1525), 1815-1830.

Vogels, J., Krahmer, E., \& Maes, A. (2015). How cognitive load influences speakers' choice of referring expressions. Cognitive science, 39(6), 1396-1418.

Weiss-Cohen, L., Konstantinidis, E., Speekenbrink, M., \& Harvey, N. (2018). Task complexity moderates the influence of descriptions in decisions from experience. Cognition, 170, 209-227.

White, P. A. (2018). Is conscious perception a series of discrete temporal frames?. Consciousness and cognition, 60, 98-126.

Xin, Y., Xu, P., Aleman, A., Luo, Y., \& Feng, T. (2020). Intrinsic prefrontal organization underlies associations between achievement motivation and delay discounting. Brain Structure and Function, 225(2), 511-518.

Yantis, S., \& Jonides, J. (1984). Abrupt visual onsets and selective attention: evidence from visual search. Journal of Experimental Psychology: Human Perception and Performance, 16(1), 121-134.

Yantis, S., \& Jonides, J. (1990). Abrupt visual onsets and selective attention: voluntary versus automatic allocation. Journal of Experimental Psychology: Human Perception and Performance, 16(1), 121-134.

Zhang, S., \& Feng, T. (2019). Modeling procrastination: Asymmetric decisions to act between the present and the future. Journal of Experimental Psychology: General. 149(2), 311-322. 